\title{
From Application to Evaluation: Addressing the Usefulness of Resilience and Vulnerability
}

\author{
Alexander Fekete • Gabriele Hufschmidt
}

Published online: 29 March 2014

(c) The Author(s) 2014. This article is published with open access at Springerlink.com

What are the benefits of the resilience and vulnerability concepts for disaster risk management? This question goes beyond the previous stage of enquiring what exactly both terms and concepts mean, how they can be measured or applied. We aim to demonstrate the potential of applied resilience and vulnerability research for disaster risk management (DRM), while addressing accompanying challenges.

This special issue grew out of the idea to generate an overview on the state-of-the-art of resilience and vulnerability studies in Germany, Austria, and Switzerland. In the past years we have experienced a huge demand from our students as well as academic and professional colleagues for an overview of studies of and experiences with resilience or vulnerability within a specific hazard, country, methodological or other context. Based on this we developed the idea to compile and structure the existing state-ofthe-art within what we termed an "Atlas of Vulnerability and Resilience Research," aiming at promoting different types of knowledge, knowledge transfer between academic disciplines and between science, policy, and practice, and underlining the role of education, learning, and communication. In order to prepare such a compendium we started to spread the idea and build up a network.

As a first milestone, in November 2012 we were invited by the German disaster network (KatNet-Katastrophennetzwerk) to jointly conduct a symposium on the introductory

\footnotetext{
A. Fekete $(\square)$

Risk and Crisis Management Section, Cologne University of Applied Sciences, 50679 Cologne, Germany

e-mail: alexander.fekete@fh-koeln.de

G. Hufschmidt

Department of Geography, Master Program Disaster

Management and Risk Governance, University of Bonn,

53115 Bonn, Germany
}

question. Around 100 participants from social and natural science disciplines and practitioners from the private sector, civil protection, and emergency relief organizations came together. Some of the key outcomes of that symposium are documented here for an international audience, thanks to the International Journal of Disaster Risk Science.

The authors of this special issue cover different concepts, methods, and experiences. They offer a look into recent concepts and applied studies in various disciplines. In addition to six research articles, two short essays offer provocative and alternative views on individual aspects of benefits and challenges that characterize resilience and vulnerability research and application.

Within this special issue it is not possible to adequately answer the introductory question, neither can the selected articles represent even the core range of disciplines, fields, concepts, methods, countries, or research contexts of resilience and vulnerability in DRM. Rather, the articles highlight some important issues, and share a discussion of the potential benefits and challenges of vulnerability and resilience for DRM as well as common themes: the appreciation of different types of knowledge, knowledge transfer, education, learning, and communication.

The article with our co-author Sylvia Kruse focuses on how the concepts of resilience and vulnerability are used in science, policy, and practice. We aim to identify and systemize some benefits and challenges and address the need to use criteria of how the benefits of both concepts for DRM can be evaluated. The article provides a background for the reasons to compile such a special issue, and discusses the roles of resilience and vulnerability in science and their usage in different European and UN policy fields. A survey of the KatNet symposium participants reflects some opinions on the benefits and challenges of the concepts of resilience and vulnerability. 
Typical for many resilience and vulnerability studies and concepts is a prognostic view. Hagemeier-Klose, Beichler, Davidse, and Deppisch adapt a "scenario planning process" and "the dynamic knowledge loop" as an innovative analysis tool. In their view, establishing communication and social learning is promoted by participatory processes and intensive science-practice cooperation that are effective when repetitive and dynamic. The authors work within the context of climate change adaptation (CCA) and underline the appreciation of learning, local types of knowledge, and knowledge transfer.

Martha Höfler assesses learning and teaching research fundamentals to create psychological resilience-building strategies in adult education. While learning is highly context-specific, relational, and dynamic, the good news is that psychological resilience can be improved, even later in life. Especially in relation to crisis and disaster risk reduction this is important knowledge for applications in civil protection such as in psychological trauma recovery, or in designing disaster preparedness strategies and concepts.

Reichel and Frömming present a case study in the Alps utilizing participatory GIS. They aim at mapping sustainable environmental knowledge, which they identify as a significant part of "cultural memory." Such knowledge is one of several types of knowledge and communicated to the interdisciplinary community of disaster risk science and management based on the participatory approach. Much more than solving just technical matters, the authors are interested in knowledge, learning, and identity. They look into how phenomena like climate change can become meaningful at the local level.

Khazai, Kunz-Plapp, Büscher, and Wegner focus on a tool (a wiki) to systematize the semantics of resilience and vulnerability, terms that are prominent for their fuzziness and resistance to unified language. A wiki is a platform to sort, unify, and share the increasing body of knowledge. There are many benefits for DRM addressed in this article and one key challenge is the sustainability or longevity of such a tool. As any other tool it will prosper based on several criteria-accessibility, usability, and continuous input from and usage by the DRM community.

Heesen, Lorenz, Nagenborg, Wenzel, and Voss investigate challenges of mapping which, just like any other method, represents a model of reality. However, since maps are visual, they look convincing and tempt users to misinterpret their presentation as reality. While this is not different in general from how much impact pictures, numbers, or phrases and narratives have on public opinion, decision-makers, and scientists, it is important to outline the usefulness and limitations of maps since they are common tools in DRM. This assessment addresses another core aspect: resilience and vulnerability in a prognostic understanding produce hypothetical information about potential risks or developments. But as soon as we see a red area on a map, do we already take this as reality, or are we aware that it is just a scientific hypothesis?

The short article by Taubenböck and Geiß is a commentary that explicitly addresses some of the questions very often heard within our community, urging us to stop the terminological and conceptual debates and move further towards application. We might then ponder if the authors are right-should we stop investigations about theory and concepts? Or, is the discussion about why we do what we do and how this can be generalized not a hallmark of science-even in the age of "big data"? With such commentary we wish to encourage further debate and meetings, especially between social and natural scientists, researchers, and practitioners.

The short article by Fekete and Sakdapolrak addresses "loss and damage" as an emerging discussion and policy field in the climate change community. Is this already the debate that will engulf the community beyond resilience and vulnerability? Is it a new turn after a long period of stressing the importance of prevention, preparedness, and prognostics? Such statements are meant to provoke discussion.

Recent disasters that attract global attention are often related to climate change discussions. Whether this relation is always justified and scientifically sound, considering existing uncertainties in science, is not followed up in this special issue. However, such extreme events and climate change adaptation (CCA) might be the most global problems that involve DRM and the questions which concepts are best suited to tackle such problems. On the background of the Intergovernmental Panel on Climate Change (IPCC) negotiations that often seem stalled, or the Hyogo Framework for Action that is turning ten in 2015, we ask in this issue:

What are the benefits of the resilience and vulnerability concepts? What alternatives could other concepts offer, such as adaptation, loss and damage, or sustainability? What are the "known knowns" and what are the "known unknowns" of resilience and vulnerability? For instance, comparable to so-called "mal-adaptation," is there an equivalent field of evaluation of mal-resilience or malvulnerability practices?

The articles in this special issue address these questions and feature current applications of resilience and vulnerability research, practice, and policy with relevance to disaster risk management. Some of the benefits and challenges associated with resilience and vulnerability are discussed, while keeping a close connection to both research and practice.

Open Access This article is distributed under the terms of the Creative Commons Attribution License which permits any use, distribution, and reproduction in any medium, provided the original author(s) and the source are credited. 